\title{
THE SOLUTION OF SOME INTEGRAL EQUATIONS
}

\author{
JOHN F. AHNER ${ }^{1}$ AND JOHN S. LOWNDES ${ }^{2}$
}

(Received 28 April 1986; revised 15 January 1987)

\begin{abstract}
Algorithms are developed by means of which certain connected pairs of Fredholm integral equations of the first and second kinds can be converted into Fredholm integral equations of the second kind. The methods are then used to obtain the solutions of two different sets of triple integral equations that occur in mixed boundary value problems involving Laplace's equation and the wave equation respectively.
\end{abstract}

\section{Introduction}

In two previous papers [1], [2] we have discussed certain classes of Fredholm integral equations of the first kind that arise in the investigation of solutions to some dual-triple-quadruple integral and series equations. In those papers we presented constructive methods for reformulating the integral equations as. Fredholm integral equations of the second kind from which the solutions of the original equations can be found.

In this paper we extend the discussion to another class of linked pairs of integral equations of the first and second kinds which occur naturally in the investigation of some three-part boundary value problems in which Dirichlet, Neumann and Robin-type boundary conditions are imposed on the three different portions of the boundary.

As a simple illustrative example, consider the problem of finding the axially symmetric potential function $\phi(r, z)$ which satisfies the equation

$$
\frac{1}{r} \frac{\partial}{\partial r}\left(r \frac{\partial \phi}{\partial r}\right)+\frac{\partial^{2} \phi}{\partial z^{2}}=0, \quad r>0, z>0,
$$

\footnotetext{
${ }^{1}$ Department of Mathematics, Vanderbilt University, Nashville, Tennessee 37235, U.S.A.

${ }^{2}$ Department of Mathematics, University of Strathclyde, Glasgow G1 1XH, U.K.

(1) Copyright Australian Mathematical Society 1987, Serial-fee code 0334-2700/87
} 
the mixed boundary conditions

$$
\begin{gathered}
\phi(r, 0)=f(r), \quad 0<r<a ; \quad \phi_{z}(r, 0)=-g(r), \quad a<r<b ; \\
\phi(r, 0)-p \phi_{z}(r, 0)=h(r), \quad b<r<\infty
\end{gathered}
$$

and is such that $\phi \rightarrow 0$ as $r^{2}+z^{2} \rightarrow \infty$.

It can be shown that a suitable form for the solution of the problem is given by

$$
\phi(r, z)=\int_{0}^{\infty} A(\xi) e^{-\xi z} J_{0}(\xi r) d \xi,
$$

where the function $A(\xi)$ is such that the integral in (1.3) exists as an improper integral and that it tends to zero as $r^{2}+z^{2} \rightarrow \infty$. Furthermore, from the boundary conditions (1.2), the unknown $A(\xi)$ must satisfy the following set of triple integral equations

$$
\begin{gathered}
\int_{0}^{\infty} A(\xi) J_{0}(\xi r) d \xi=f(r), \quad 0<r<a, \\
\int_{0}^{\infty} \xi A(\xi) J_{0}(\xi r) d \xi=g(r), \quad a<r<b, \\
\int_{0}^{\infty}(1+p \xi) A(\xi) J_{0}(\xi r) d \xi=h(r), \quad b<r<\infty .
\end{gathered}
$$

Triple integral equations of this type do not seem to have been treated in the literature (ci. the integral equations discussed by Sneddon [6]).

We shall show that the problem of solving such sets of equations can be trantsformed into that of solving a connected pair of Fredholm integral equations of the first and second kinds of the type

$$
\begin{gathered}
\rho(x) f_{1}(x)+\left\{\int_{a}^{b} f_{1}(y)+\int_{c}^{d} f_{2}(y)\right\} K(x, y) d y=g(x), \quad 0 \leqslant a<x<b, \\
r(x) f_{2}(x)+\left\{\int_{a}^{b} f_{1}(y)+\int_{c}^{d} f_{2}(y)\right\} K(x, y) d y=h(x), \quad c<x<d,
\end{gathered}
$$

where $b \leqslant c$.

In the above equations $g(x), h(x), r(x)$ and $\rho(x)$ are known functions, $f_{1}(x)$ and $f_{2}(x)$ are the solution functions to be determined and the kernel function can take either of the forms

$$
\begin{aligned}
& K(x, y)=L_{o}(x, y: a), \quad \sigma=\min (x, y), \\
& K(x, y)=M_{\lambda}(x, y: d), \quad \lambda=\max (x, y),
\end{aligned}
$$

where the precise definitions of $L_{\sigma}$ and $M_{\lambda}$ will be given in Sections 2 and 3, respectively. 
In Sections 2 and 3 we show that the problem of solving the pair of equations (1.7) and (1.8) can, in each case, be reduced to that of solving a Fredholm integral equation of the second kind. To illustrate the use of the algorithms developed in these sections we then solve, in Section 4, some general triple integral equations which include equations (1.4) to (1.6) as a special case and in Section 5 we discuss some triple integral equations that arise in mixed boundary value problems involving the wave equation.

\section{Integral equations with the kernel $L_{\sigma}(x, y: 0)$}

We first of all consider the problem of finding the solution functions $f_{1}(x)$ and $f_{2}(x)$ which satisfy the pair of integral equations

$$
\begin{gathered}
\left\{\int_{0}^{a} f_{1}(y)+\int_{b}^{c} f_{2}(y)\right\} L_{\sigma}(x, y: 0) d y=g(x), \quad 0<x<a, \quad(2.1) \\
\left\{\int_{0}^{a} f_{1}(y)+\int_{b}^{c} f_{2}(y)\right\} L_{\mathrm{o}}(x, y: 0) d y+r(x) f_{2}(x)=h(x), \quad b<x<c,
\end{gathered}
$$

where $a \leqslant b, \sigma=\min (x, y), g(x), h(x)$ and $r(x)$ are given functions such that $r(x) \neq 0, x \in[b, c]$ and in general the kernel function takes the form

$$
L_{\beta}(x, y: \alpha)=\int_{\alpha}^{\beta} \phi(t) p_{1}(t, x) p_{2}(t, y) d t, \quad 0 \leqslant \alpha<\beta \leqslant c .
$$

In the above definition $\phi(t)$ is a given non-zero function and the $p_{i}(t, x)$, $i=1,2$ are the known kernel functions of the integral operators

$$
\begin{gathered}
P_{i}(\gamma, \delta) f(x)=\int_{\gamma}^{\delta} p_{i}(t, x) f(t) d t, \\
P_{i}^{*}(\gamma, \delta) f(x)=\int_{\gamma}^{\delta} p_{i}(x, t) f(t) d t, \quad 0 \leqslant \gamma \leqslant x \leqslant \delta \leqslant c,
\end{gathered}
$$

which are assumed to have unique inverses denoted by $\left(P_{i}(\gamma, \delta)\right)^{-1}$ and $\left(P_{i}^{*}(\gamma, \delta)\right)^{-1}$, respectively.

With the above definition for $L_{\sigma}(x, y: 0)$ we see that $(2.1)$ and $(2.2)$ can be written as

$$
\begin{gathered}
\left\{\int_{0}^{x} L_{y}(x, y: 0)+\int_{x}^{a} L_{x}(x, y: 0)\right\} f_{1}(y) d y+\int_{b}^{c} L_{x}(x, y: 0) f_{2}(y) d y=g(x) \\
0<x<a
\end{gathered}
$$


On inverting the orders of the integrations in these equations and using the definitions (2.4) we find that they can be expressed in the operational forms

$$
\begin{gathered}
P_{1}(0, x) \phi(x)\left[P_{2}^{*}(x, a) f_{1}(x)+P_{2}^{*}(b, c) f_{2}(x)\right]=g(x), \quad 0<x<a, \\
P_{1}(0, a) \phi(x) P_{2}^{*}(x, a) f_{1}(x)+P_{1}(0, b) \phi(x) P_{2}^{*}(b, c) f_{2}(x) \\
\quad+P_{1}(b, x) \phi(x) P_{2}^{*}(x, c) f_{2}(x)+r(x) f_{2}(x)=h(x), \quad b<x<c .
\end{gathered}
$$

To avoid any possible confusion with our notation, it follows from the definitions (2.4) that the first expression appearing on the left hand side of (2.7) is given by

$$
P_{1}(0, x) \phi(x) P_{2}^{*}(x, a) f_{1}(x)=\int_{0}^{x} p_{1}(t, x) \phi(t)\left(\int_{t}^{a} p_{2}(t, y) f_{1}(y) d y\right) d t .
$$

A similar interpretation holds for the second term in (2.7) as well as the various integral operators in (2.8).

From (2.7) we have that

$$
\phi(x) P_{2}^{*}(x, a) f_{1}(x)=\left(P_{1}(0, x)\right)^{-1} g(x)-\phi(x) P_{2}^{*}(b, c) f_{2}(x), \quad 0<x<a
$$

and eliminating $\phi(x) P_{2}^{*}(x, a) f_{1}(x)$ between this equation and (2.8) we obtain, after some simplification, the equation

$$
\begin{array}{r}
P_{1}(a, b) \phi(x) P_{2}^{*}(b, c) f_{2}(x)+P_{1}(b, x) \phi(x) P_{2}^{*}(x, c) f_{2}(x) \\
+r(x) f_{2}(x)=G(x), \quad b<x<c,
\end{array}
$$

where $G(x)$ is the known function

$$
G(x)=h(x)-P_{1}(0, a)\left(P_{1}(0, x)\right)^{-1} g(x) .
$$

Inverting the orders of the integrations in (2.10) and using (2.3) we arrive at the following Fredholm integal equation of the second kind for the determination of $f_{2}(x)$

$$
r(x) f_{2}(x)+\int_{b}^{c} L_{\sigma}(x, y: a) f_{2}(y) d y=G(x), \quad b<x<c .
$$

When $f_{2}(x)$ has been found from this equation the solution function $f_{1}(x)$ can be evaluated from (2.9) in the form

$$
\begin{gathered}
f_{1}(x)=\left(P_{2}^{*}(x, a)\right)^{-1}\left[\frac{1}{\phi(x)}\left(P_{1}(0, x)\right)^{-1} g(x)-P_{2}^{*}(b, c) f_{2}(x)\right], \\
0<x<a .
\end{gathered}
$$


3. Integral equations with the kernel $M_{\lambda}(x, y: d)$

We next consider the pair of integral equations

$$
\begin{array}{r}
\rho(x) u_{1}(x)+\left\{\int_{a}^{b} u_{1}(y)+\int_{c}^{d} u_{2}(y)\right\} M_{\lambda}(x, y: d) d y=g(x) \\
0 \leqslant a<x<b \\
\left\{\int_{a}^{b} u_{1}(y)+\int_{c}^{d} u_{2}(y)\right\} M_{\lambda}(x, y: d) d y=h(x), \quad c<x<d,
\end{array}
$$

where $b \leqslant c, \lambda=\max (x, y), u_{1}(x)$ and $u_{2}(x)$ are the required solution functions and $g(x), h(x)$ and $\rho(x)$, where $\rho(x) \neq 0, x \in[a, b]$, are prescribed functions.

In general the kernel function $M_{\lambda}(x, y: d)$ is defined by

$$
M_{\alpha}(x, y: \beta)=\int_{\alpha}^{\beta} \psi(t) p_{1}(x, t) p_{2}(y, t) d t, \quad 0 \leqslant \alpha<\beta \leqslant d,
$$

where $\psi(t)$ is a given non-zero function and $p_{i}(x, t), i=1,2$ are the known kernel functions of the integral operators defined by (2.4). It will be noted that the arguments of the functions $p_{i}$ in (3.3) are transposed with those in (2.3); however, we can still use the same definitions (2.4) for the integral operators $P_{i}$ and $P_{i}^{*}$.

With the above definition for $M_{\lambda}(x, y: d), \lambda=\max (x, y)$, we can invert the orders of the integrations in (3.1) and (3.2) and use the definitions (2.4) to find that the pair of equations can be written in the operational forms

$$
\begin{gathered}
\rho(x) u_{1}(x)+P_{1}^{*}(x, b) \psi(x) P_{2}(a, x) u_{1}(x)+P_{1}^{*}(b, d) \psi(x) P_{2}(a, b) u_{1}(x) \\
+P_{1}^{*}(c, d) \psi(x) P_{2}(c, x) u_{2}(x)=g(x), \quad a<x<b, \\
P_{1}^{*}(x, d) \psi(x)\left[P_{2}(a, b) u_{1}(x)+P_{2}(c, x) u_{2}(x)\right]=h(x), \quad c<x<d .
\end{gathered}
$$

Solving (3.5) for $\psi(x) P_{2}(c, x) u_{2}(x)$ and substituting the result into (3.4), we obtain, after a little simplification, the equation

$$
\begin{aligned}
\rho(x) u_{1}(x) & +P_{1}^{*}(x, b) \psi(x) P_{2}(a, x) u_{1}(x) \\
& +P_{1}^{*}(b, c) \psi(x) P_{2}(a, b) u_{1}(x)=\tilde{G}(x), \quad a<x<b
\end{aligned}
$$

where

$$
\tilde{G}(x)=g(x)-P_{1}^{*}(c, d)\left(P_{1}^{*}(x, d)\right)^{-1} h(x)
$$

is a known function. 
Interchanging the orders of the integrations in (3.6) and using (3.3), we get the following Fredholm integral equation of the second kind which determines the function $u_{1}(x)$ :

$$
\rho(x) u_{1}(x)+\int_{a}^{b} M_{\lambda}(x, y: c) u_{1}(y) d y=\tilde{G}(x), \quad a<x<b .
$$

When $u_{1}(x)$ has been found from this equation the solution function $u_{2}(x)$ can be found from (3.5) when written in the form

$$
\begin{array}{r}
u_{2}(x)=\left(P_{2}(c, x)\right)^{-1}\left[\frac{1}{\psi(x)}\left(P_{1}^{*}(x, d)\right)^{-1} h(x)-P_{2}(a, b) u_{1}(x)\right], \\
c<x<d .
\end{array}
$$

\section{Triple integral equations involving the Hankel transform}

As an application of the algorithm described in Section 2 we consider the following general set of triple integral equations

$$
\begin{aligned}
S_{\frac{1}{2} \mu-\alpha, 2 \alpha} A(x) & =0, & 0<x<a, \\
S_{\frac{1}{2} \nu-\beta, 2 \beta} A(x) & =g(x), & a<x<b, \\
S_{\frac{1}{2} \mu-\alpha, 2 \alpha} A(x)+k S_{\frac{1}{2} \nu-\beta, 2 \beta} A(x) & =0, & b<x<\infty,
\end{aligned}
$$

where $\mu, \nu, \alpha, \beta$ and $k$ are real constants such that $2(\alpha-\beta) \geqslant|\mu-\nu|, g(x)$ is a prescribed function, $A(x)$ is the solution function to be determined and

$$
S_{\eta, \alpha} f(x)=2^{\alpha} x^{-\alpha} \int_{0}^{\infty} t^{1-\alpha} J_{2 \eta+\alpha}(x t) f(t) d t
$$

denotes the modified operator of the Hankel transform [6] with the inversion formula

$$
S_{\eta, \alpha}^{-1}=S_{\eta+\alpha,-\alpha}
$$

With $f=h=0$ and $p=(k r) / 2$ it is easily seen that the triple integral equations (1.4) to (1.6) are a special case of (4.1) to (4.3) when $\alpha=\frac{1}{2}$ and $\beta=\mu=\nu=0$.

In order to reduce the above triple integral equations to a connected pair of Fredholm integral equations of the first and second kinds we introduce two, as yet unknown, functions $f_{1}(x)$ and $f_{2}(x)$ defined by the equations

$$
\begin{aligned}
S_{\frac{1}{2} \nu-\beta, 2 \beta} A(x) & =f_{1}(x), & & 0<x<a, \\
& =f_{2}(x), & & b<x<\infty .
\end{aligned}
$$


Applying the inversion formula (4.5) to (4.2) and (4.6) we find that

$$
A(x)=2^{-2 \beta} x^{2 \beta}\left\{\int_{0}^{a} f_{1}(y)+\int_{a}^{b} g(y)+\int_{b}^{\infty} f_{2}(y)\right\} y^{1+2 \beta} J_{\nu}(x y) d y .
$$

On substituting this expression for $A(x)$ into (4.1) and (4.3) and interchanging the orders of the integrations, we find that the equations take on the forms

$$
\begin{gathered}
\left\{\int_{0}^{a} f_{1}(y)+\int_{b}^{\infty} f_{2}(y)\right\} L_{\mathrm{o}}^{(1)}(x, y: 0) d y=\tilde{g}_{1}(x), \quad 0<x<a, \quad(4.8) \\
\left\{\int_{0}^{a} f_{1}(y)+\int_{b}^{\infty} f_{2}(y)\right\} L_{\sigma}^{(1)}(x, y: 0) d y+k f_{2}(x)=\tilde{g}_{2}(x), \quad b<x<\infty,
\end{gathered}
$$

where

$$
\tilde{g}_{1}(x)=-\int_{a}^{b} g(y) L_{x}^{(1)}(x, y: 0) d y, \quad \tilde{g}_{2}(x)=-\int_{a}^{b} g(y) L_{y}^{(1)}(x, y: 0) d y,
$$

are known functions. The kernel of the equations is given by

$$
\begin{aligned}
L_{o}^{(1)}(x, y: 0) & =2^{2(\alpha-\beta)} x^{-2 \alpha} y^{1+2 \beta} \int_{0}^{\infty} t^{1-2(\alpha-\beta)} J_{\mu}(x t) J_{\nu}(y t) d t \\
& =\frac{4 x^{-2 \alpha-\mu} y^{1+2 \beta-\nu}}{\Gamma(\mu-\gamma) \Gamma(\nu-\gamma)} \int_{0}^{\sigma} t^{1+2 \gamma}\left(x^{2}-t^{2}\right)^{\mu-\gamma-1}\left(y^{2}-t^{2}\right)^{\nu-\gamma-1} d t,
\end{aligned}
$$

where $\sigma=\min (x, y), \gamma=\frac{1}{2}(\mu+\nu)-(\alpha-\beta), 2(\alpha-\beta)>|\mu-\nu|$. The expression (4.12) is proved in the appendix and is similar to the result stated by Williams [7].

It will be seen that (4.8) and (4.9) are precisely of the same type as the pair of equations discussed in Section 2.

On comparing the definitions of the kernel functions (2.3) and (4.12) we see that we must take $\phi(t)=1$ and the kernel functions $p_{i}, i=1,2$, to be

$$
\begin{aligned}
& p_{1}(t, x)=\frac{2 x^{-\mu-2 \alpha}}{\Gamma(\mu-\gamma)}\left(x^{2}-t^{2}\right)^{\mu-\gamma-1} t^{1+\nu+2 \beta}, \\
& p_{2}(t, y)=\frac{2 t^{\mu-2 \alpha}}{\Gamma(\nu-\gamma)}\left(y^{2}-t^{2}\right)^{\nu-\gamma-1} y^{1+2 \beta-\nu} .
\end{aligned}
$$

Associated with these kernel functions we can define the integral operators

$$
\begin{aligned}
P_{1}(\varepsilon, x) f(x) & =I_{\frac{1}{2} \nu+\beta, \mu-\gamma}(\varepsilon, x) f(x), \\
P_{1}^{*}(x, \delta) f(x) & =x K_{\frac{1}{2} \nu+\beta, \mu-\gamma}(x, \delta) x^{-1} f(x), \\
P_{2}(\varepsilon, x) f(x) & =x I_{\frac{1}{2} \mu-\alpha, \nu-\gamma}(\varepsilon, x) x^{-1} f(x), \\
P_{2}^{*}(x, \delta) f(x) & =K_{\frac{1}{2} \mu-\alpha, \nu-\gamma}(x, \delta) f(x),
\end{aligned}
$$

where $\gamma=\frac{1}{2}(\mu+\nu)-(\alpha-\beta)$. 
In the above expressions $I_{\eta, \alpha}$ and $K_{\eta, \alpha}$ are the modified Erdélyi-Kober operators of fractional integration which are defined in [4] by

$$
\begin{aligned}
I_{\eta, \alpha}(\varepsilon, x) f(x) & =\frac{2 x^{-2(\alpha+\eta)}}{\Gamma(\alpha)} \int_{\varepsilon}^{x}\left(x^{2}-t^{2}\right)^{\alpha-1} t^{1+2 \eta} f(t) d t, \quad \alpha>0, \quad(4.19) \\
& =x^{-1-2(\alpha+\eta)} \mathscr{D}_{x}^{s}\left\{x^{1+2(\alpha+\eta+s)} I_{\eta, \alpha+s}(\varepsilon, x) f(x)\right\}, \quad \alpha<0, \\
K_{\eta, \alpha}(x, \delta) f(x) & =\frac{2 x^{2 \eta}}{\Gamma(\alpha)} \int_{x}^{\delta}\left(t^{2}-x^{2}\right)^{\alpha-1} t^{1-2(\alpha+\eta)} f(t) d t, \quad \alpha>0, \quad(4.21) \\
& =(-1)^{s} x^{-1+2 \eta \mathscr{D}_{x}^{s}}\left\{x^{1+2(s-\eta)} K_{\eta-s, \alpha+s}(x, \delta) f(x)\right\}, \quad \alpha<0,
\end{aligned}
$$

where

$$
\mathscr{D}_{x}=\frac{1}{2} \frac{d}{d x} \frac{1}{x}
$$

and $s$ is a positive integer such that $0<\alpha+s<1$, when $\alpha<0$.

The inverse operators are defined by

$$
I_{\eta, \alpha}^{-1}(\varepsilon, x)=I_{\eta+\alpha,-\alpha}(\varepsilon, x), \quad K_{\eta, \alpha}^{-1}(x, \delta)=K_{\eta+\alpha,-\alpha}(x, \delta) .
$$

From the above definitions it follows that the integral operators (4.15) to (4.18) possess the inverses given by

$$
\begin{aligned}
\left(P_{1}(\varepsilon, x)\right)^{-1} f(x) & =I_{\frac{1}{2} \mu+\alpha, \gamma-\mu}(\varepsilon, x) f(x), \\
\left(P_{1}^{*}(x, \delta)\right)^{-1} f(x) & =x K_{\frac{1}{2} \mu+\alpha, \gamma-\mu}(x, \delta) x^{-1} f(x), \\
\left(P_{2}(\varepsilon, x)\right)^{-1} f(x) & =x I_{\frac{1}{2} \nu-\beta, \gamma-\nu}(\varepsilon, x) x^{-1} f(x), \\
\left(P_{2}^{*}(x, \delta)\right)^{-1} f(x) & =K_{\frac{1}{2} \nu-\beta, \gamma-\nu}(x, \delta) f(x) .
\end{aligned}
$$

Using the above results and the general method of solution given in Section 2, we find from (2.12) with $r(x) \equiv k$, that the function $f_{2}(x)$ which satisfies the pair of equations (4.8) and (4.9) is the solution of the Fredholm integral equation of the second kind

$$
k f_{2}(x)+\int_{b}^{\infty} f_{2}(y) L_{\sigma}^{(1)}(x, y: a) d y=G(x), \quad b<x<\infty
$$

with

$$
L_{\sigma}^{(1)}(x, y: a)=\int_{a}^{\sigma} p_{1}(t, x) p_{2}(t, y) d t, \quad \sigma=\min (x, y)
$$


where the $p_{i}(t, x), i=1,2$, are given by (4.13) and (4.14) and the free term is the known function

$$
G(x)=\tilde{g}_{2}(x)-I_{\frac{1}{2} \nu+\beta, \mu-\gamma}(0, a) I_{\frac{1}{2} \mu+\alpha, \gamma-\mu}(0, x) \tilde{g}_{1}(x)
$$

where $\tilde{g}_{1}(x)$ and $\tilde{g}_{2}(x)$ are defined by (4.10).

Once $f_{2}(x)$ has been found from the above integral equation we have, on using (2.13), that $f_{1}(x)$ is given by

$$
\begin{aligned}
& f_{1}(x)=K_{\frac{1}{2} \nu-\beta, \gamma-\nu}(x, a)\left[I_{\frac{1}{2} \mu+\alpha, \gamma-\mu}(0, x) \tilde{g}_{1}(x)\right. \\
& \left.-K_{\frac{1}{2} \mu-\alpha, \nu-\gamma}(b, \infty) f_{2}(x)\right], \\
& 0<x<a \text {. }
\end{aligned}
$$

Finally, having determined $f_{1}(x)$ and $f_{2}(x)$, we see that the solution function $A(x)$ of the triple integral equations (4.1) to (4.3) is given by (4.7).

\section{Triple integral equations involving the Kontorovich-Lebedev transform}

To illustrate an application of the general method given in Section 3 we shall consider an extension of the triple integral equations solved in [3], special cases of which occur in three-part boundary value problems involving the wave equation. These are the equations

$$
\begin{gathered}
\quad \frac{2}{\pi^{2}} \int_{0}^{\infty} t \sinh (\pi t) A(t) K_{i t}(p x) d t=0, \quad 0<x<a, \\
\frac{2 k}{\pi^{2}} \int_{0}^{\infty} t \sinh (\pi t) A(t) K_{i t}(p x) d t \\
+\frac{2}{\pi^{2}} \int_{0}^{\infty} t \sinh (2 \pi t) \Gamma(\alpha+i t) \Gamma(\alpha-i t) A(t) K_{i t}(p x) d t=s(x), \\
\quad a<x<b, \quad(5.2) \\
\frac{2}{\pi^{2}} \int_{0}^{\infty} t \sinh (2 \pi t) \Gamma(\alpha+i t) \Gamma(\alpha-i t) A(t) K_{i t}(p x) d t=0, \quad b<x<\infty
\end{gathered}
$$

where $0 \leqslant a<b<\infty, 0 \leqslant 2 \alpha<1, k$ is a constant, $s(x)$ is a prescribed function, $K_{i t}(p x), \operatorname{Re}(p)>0$, is the modified Bessel (Macdonald) function and $A(t)$ is the solution function to be determined.

Two results which' we shall find useful are as follows. The Kontorovich-Lebedev transform of the function $f(y), 0 \leqslant y<\infty$, is defined by

$$
F(t)=\int_{0}^{\infty} y^{-1} f(y) K_{i t}(p y) d y, \quad \operatorname{Re}(p)>0,
$$


with the inversion formula

$$
f(y)=\frac{2}{\pi^{2}} \int_{0}^{\infty} t \sinh (\pi t) F(t) K_{i t}(p y) d t .
$$

A transform result is

$$
\begin{aligned}
& \frac{2}{\pi^{2}} \int_{0}^{\infty} t \sinh (2 \pi t) \Gamma(\alpha+i t) \Gamma(\alpha-i t) K_{i t}(p x) K_{i t}(p y) d t \\
& \quad=\frac{2 \pi}{\left[\Gamma\left(\frac{1}{2}-\alpha\right)\right]^{2}}(x y)^{\alpha} e^{p(x+y)} M_{\lambda}^{(1)}(x, y: \infty), \quad 0 \leqslant 2 \alpha<1, \operatorname{Re}(p)>0,
\end{aligned}
$$

where

$$
M_{\lambda}^{(1)}(x, y: \infty)=\int_{\lambda}^{\infty} \frac{e^{-2 p t}}{[(t-x)(t-y)]^{\alpha+(1 / 2)}} d t, \quad \lambda=\max (x, y) .
$$

We now show that the set of equations (5.1) to (5.3) can be converted into a pair of Fredholm integral equations of the type considered in Section 3.

To this end we introduce two functions $u_{1}(x)$ and $u_{2}(x)$ defined by the equations

$$
\begin{aligned}
\frac{2}{\pi^{2}} \int_{0}^{\infty} t \sinh (\pi t) A(t) K_{i t}(p x) d t & =x^{1-\alpha} e^{-p x} u_{1}(x), & & a<x<b \\
& =x^{1-\alpha} e^{-p x} u_{2}(x), & & b<x<\infty
\end{aligned}
$$

and from (5.1), (5.8) and (5.9), we find, on using the reciprocal formulae (5.4) and (5.5), that the function $A(t)$ is given by

$$
A(t)=\left\{\int_{a}^{b} u_{1}(y)+\int_{b}^{\infty} u_{2}(y)\right\} y^{-\alpha} e^{-p y} K_{i t}(p y) d y .
$$

After substituting this expression for $A(t)$ into (5.2) and (5.3), inverting the orders of the integrations and making use of the results (5.6) and (5.7), we see that they become the pair of integral equations

$$
\begin{aligned}
& \rho_{1}(x) u_{1}(x)+\left\{\int_{a}^{b} u_{1}(y)+\int_{b}^{\infty} u_{2}(y)\right\} M_{\lambda}^{(1)}(x, y: \infty) d y \\
& =\frac{\left[\Gamma\left(\frac{1}{2}-\alpha\right)\right]^{2}}{2 \pi} x^{-\alpha} e^{-p x} s(x), \quad a<x<b, \\
& \left\{\int_{a}^{b} u_{1}(y)+\int_{b}^{\infty} u_{2}(y)\right\} M_{\lambda}^{(1)}(x, y: \infty) d y=0, \quad b<x<\infty,
\end{aligned}
$$


where

$$
\rho_{1}(x)=\frac{k}{2 \pi}\left[\Gamma\left(\frac{1}{2}-\alpha\right)\right]^{2} x^{1-2 \alpha} e^{-p x}
$$

and these are the type of integral equations discussed in Section 3.

Using the general results of that section and taking $\psi(t)=e^{-2 p t}, p_{1}(x, t)=$ $p_{2}(x, t)=(t-x)^{-(\alpha+1 / 2)}$, it follows that the kernel function is

$$
M_{\lambda}^{(1)}(x, y: \infty)=\int_{\lambda}^{\infty} \psi(t) p_{1}(x, t) p_{2}(y, t) d t, \quad \lambda=\max (x, y)
$$

Associated with the functions $p_{i}, i=1,2$, we have the Abel-type integral operators

$$
\begin{array}{cc}
P(\gamma, x) f(x)=\int_{\gamma}^{x}(x-t)^{-(\alpha+1 / 2)} f(t) d t, & 0 \leqslant 2 \alpha<1, \\
P^{*}(x, \delta) f(x)=\int_{x}^{\delta}(t-x)^{-(\alpha+1 / 2)} f(t) d t, & 0 \leqslant 2 \alpha<1,
\end{array}
$$

which possess the inverses

$$
\begin{gathered}
(P(\gamma, x))^{-1} f(x)=\frac{\cos (\alpha \pi)}{\pi} \frac{d}{d x} \int_{\gamma}^{x}(x-t)^{\alpha-1 / 2} f(t) d t \\
\left(P^{*}(x, \delta)\right)^{-1} f(x)=-\frac{\cos (\alpha \pi)}{\pi} \frac{d}{d x} \int_{x}^{\delta}(t-x)^{\alpha-1 / 2} f(t) d t
\end{gathered}
$$

Following Section 3, we find on using the above results and (3.8), that the required solution function $u_{1}(x)$ can be found from the following Fredholm integral equation of the second kind

$$
\begin{gathered}
\rho_{1}(x) u_{1}(x)+\int_{a}^{b} M_{\lambda}^{(1)}(x, y: b) u_{1}(y) d y=\frac{\left[\Gamma\left(\frac{1}{2}-\alpha\right)\right]^{2}}{2 \pi} x^{-\alpha} e^{-p x} s(x), \\
a<x<b,
\end{gathered}
$$

where $\rho_{1}(x)$ is given by $(5.13)$ and

$$
M_{\lambda}^{(1)}(x, y: b)=\int_{\lambda}^{b} \frac{e^{-2 p t}}{[(t-x)(t-y)]^{\alpha+(1 / 2)}} d t, \quad \lambda=\max (x, y) .
$$

When $u_{1}(x)$ has been determined, we see from (3.9) that the second solution function can be calculated from the equation

$$
\begin{aligned}
u_{2}(x) & =-(P(b, x))^{-1} P(a, b) u_{1}(x) \\
& =-\frac{\cos (\alpha \pi)}{\pi} \frac{d}{d x} \int_{b}^{x}(x-t)^{\alpha-1 / 2} d t \int_{a}^{b}(t-y)^{-(\alpha+1 / 2)} u_{1}(y) d y \\
& =-\frac{\cos (\alpha \pi)}{\pi(x-b)^{1 / 2-\alpha}} \int_{a}^{b} \frac{(b-y)^{1 / 2-\alpha}}{x-y} u_{1}(y) d y, \quad b<x<\infty .
\end{aligned}
$$


Finally, knowing $u_{1}(x)$ and $u_{2}(x)$, the solution function $A(t)$ of the triple integral equations (5.1) to (5.3) can be found from (5.10).

\section{Appendix}

THEOREM.

$$
\begin{aligned}
& \int_{0}^{\infty} t^{1+2 \gamma-(\mu+\nu)} J_{\mu}(x t) J_{\nu}(y t) d t \\
& =\frac{2^{2(\gamma+1)-\mu-\nu} x^{-\mu} y^{-\nu}}{\Gamma(\mu-\gamma) \Gamma(\nu-\gamma)} \int_{0}^{o} t^{1+2 \gamma}\left(x^{2}-t^{2}\right)^{\mu-\gamma-1}\left(y^{2}-t^{2}\right)^{\nu-\gamma-1} d t,
\end{aligned}
$$

where $\mu>\gamma, \nu>\gamma$ and $\sigma=\min (x, y)$.

Proof. In terms of the modified operator of the Hankel transform (4.4), we can express the Weber-Schafheitlin discontinuous integral [5, p. 100] in the form

$$
S_{\frac{1}{2} \gamma, 0}\left[J_{\mu}(x y) y^{\gamma-\mu}\right]=\frac{x^{-\mu} y^{\gamma}\left(x^{2}-y^{2}\right)^{\mu-\gamma-1}}{2^{\mu-\gamma-1} \Gamma(\mu-\gamma)} H(x-y),
$$

where $\mu>\gamma>-1$ and $H(x)$ denotes the Heaviside unit-function.

Operating on both sides of the above equation with the Erdélyi-Kober operator $I_{\frac{1}{2} \gamma, \nu-\gamma}(0, y)$ and making use of the result [6]

$$
I_{\eta+\alpha, \beta} S_{\eta, \alpha}=S_{\eta, \alpha+\beta}
$$

we see that it becomes

$$
\begin{aligned}
& S_{\frac{1}{2} \gamma, \nu-\gamma}\left[J_{\mu}(x y) \cdot y^{\gamma-\mu}\right] \\
& =\frac{2^{\gamma+1-\mu} x^{-\mu}}{\Gamma(\mu-\gamma)} I_{\frac{1}{2} \gamma, \nu-\gamma}(0, y)\left[y^{\gamma}\left(x^{2}-y^{2}\right)^{\mu-\gamma-1} H(x-y)\right] .
\end{aligned}
$$

When written out in detail, it is found that (A.4) and (A.1) are identical when $\nu>\gamma$ and the result of the theorem is proved.

\section{References}

[1] J. F. Ahner and J. S. Lowndes, "On the solution of a class of integral equations", J. Math. Anal. Appl. 100 (1984) 447-462.

[2] J. F. Ahner and J. S. Lowndes, "On the solution of a class of integral equations II", J. Math. Anal. Appl. 110 (1985) 391-406. 
[3] J. S. Lowndes, "On dual and triple integral equations involving modified Bessel functions", Appl. Anal. 6 (1977) 253-260.

[4] J. S. Lowndes, "The solution of some integral equations", Math. Methods Appl. Sci. 2 (1979) 26-33.

[5] W. Magnus, F. Oberhettinger and R. P. Soni, Formulas and theorems for the special functions of mathematical physics, 3rd ed. (Springer-Verlag, 1966).

[6] I. N. Sneddon, Mixed boundary value problems in potential theory (North Holland, 1966).

[7] W. E. Williams, “A class of integral equations", Proc. Camb. Phil. Soc. 59 (1963) 589-597. 